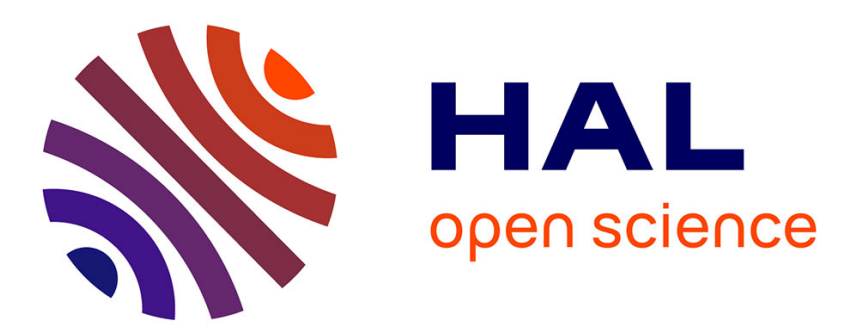

\title{
Étude de la structure des chaines dans les différentes phases, solide, nématique, ou liquide, du méthoxy benzilidène butyl aniline (MBBA)
}

\author{
C. Destrade, H. Gasparoux
}

\section{- To cite this version:}

C. Destrade, H. Gasparoux. Étude de la structure des chaines dans les différentes phases, solide, nématique, ou liquide, du méthoxy benzilidène butyl aniline (MBBA). Journal de Physique Lettres, 1975, 36 (4), pp.105-107. 10.1051/jphyslet:01975003604010500 . jpa-00231159

HAL Id: jpa-00231159

https://hal.science/jpa-00231159

Submitted on 1 Jan 1975

HAL is a multi-disciplinary open access archive for the deposit and dissemination of scientific research documents, whether they are published or not. The documents may come from teaching and research institutions in France or abroad, or from public or private research centers.
L'archive ouverte pluridisciplinaire HAL, est destinée au dépôt et à la diffusion de documents scientifiques de niveau recherche, publiés ou non, émanant des établissements d'enseignement et de recherche français ou étrangers, des laboratoires publics ou privés. 


\title{
ÉTUDE DE LA STRUCTURE DES CHAINES \\ DANS LES DIFFÉRENTES PHASES, SOLIDE, NÉMATIQUE, OU LIQUIDE, DU MÉTHOXY BENZILIDẼNE BUTYL ANILINE (MBBA)
}

\author{
C. DESTRADE et H. GASPAROUX \\ Centre de Recherche Paul-Pascal \\ Domaine Universitaire, 33405 Talence, Université de Bordeaux I, France
}

(Reçu le 21 janvier 1975, accepté le 11 février 1975)

\begin{abstract}
Résumé. - Une analyse fine des spectres Raman du MBBA dans les phases solide, nématique et liquide est proposée : on montre que ce composé présente trois formes cristallines notées A, B et $C$ et que les raies observées entre 1100 et $650 \mathrm{~cm}^{-1}$ mettent essentiellement en jeu la chaîne butyl. Les modifications importantes observées dans cette région lors des transitions de phases sont attribuées à des modifications structurales de cette chaîne; on montre en particulier que la fusion de la chaîne n'est pas totale en phase nématique et on propose une méthode de mesure directe de leur paramètre d'ordre.
\end{abstract}

\begin{abstract}
We propose a detailed analysis of MBBA Raman spectra in the solid, nematic and liquid phases. It's shown that three crystalline forms, A, B and C exist and that the Raman lines associated to the butyl chain are observed between 1100 and $650 \mathrm{~cm}^{-1}$. The strong modifications of the spectra at the phase transitions are due to structural modifications of this chain. Besides it is proved that, in the nematic phase, the melting of the chain is not complete; a method is proposed for a direct measurement of the order parameter.
\end{abstract}

Ce travail s'inscrit dans le cadre de recherches que nous développons actuellement et qui ont pour but de préciser les relations qui peuvent exister entre les propriétés macroscopiques d'une mésophase et la structure des molécules qui la composent.

Jusqu'à ces dernières années, les modèles théoriques développés pour rendre compte des propriétés physiques des mésophases [1,2] assimilaient les molécules à des bâtonnets rigides. De tels modèles se sont rapidement révélés incapables d'expliquer l'évolution des propriétés liées par exemple à l'allongement des chaînes alkyl ou alcoxy des molécules [2] [3] (*). Des travaux théoriques récents dus à Marcelja [2] ont mis en évidence la nécessité de prendre en considération la conformation des chaînes dans les modèles théoriques pour interpréter certains résultats expérimentaux.

L'influence de la longueur et de la ramification de la chaîne sur la nature de la phase obtenue étant bien établie [3] $(*)$, il est raisonnable de penser que les caractères de la transition $S_{A} \rightarrow$ Nématique, variable d'un produit à un autre, sont le reflet de modifications structurales des chaînes lorsqu'on passe d'un composé à un autre.

(*) JACQUES, J., Communication privée.
Seuls, les travaux récents de Charvolin [4] font état d'une première tentative d'analyse du comportement des chaînes dans les mésophases mais la technique utilisée (R.M.N.) ne permet pas une analyse détaillée en terme de conformation moléculaire.

Il n'existe que peu de travaux concernant l'étude d'une phase nématique par spectroscopie de vibration $[5,6,7]$.

Le but de cet article est de montrer que la spectroscopie Raman peut constituer une méthode de mesure directe de l'organisation des chaînes. Pour illustrer cette possibilité non encore exploitée, nous avons choisi de présenter les résultats obtenus sur un matériau bien connu, le MBBA.

La spectroscopie Raman a déjà été utilisée avec succès dans la mise en évidence de l'isomérie cistrans des alcanes [8]. Les résultats obtenus montrent que les vibrations de squelette de ces matériaux se traduisent par des raies fines et intenses, faciles à identifier et dont la fréquence est étroitement liée à la géométrie de la chaîne $[8,9]$.

Dans le cas du MBBA, les cycles benzéniques ne donnent pas lieu à des raies intenses dans la région $1100-650 \mathrm{~cm}^{-1}$ caractérisant les fréquences de vibration des chaînes butyl. Ce résultat a été soigneusement vérifié sur une série de $\mathrm{p}$. alkyl acétophénones. 
1. Technique expérimentale. - Le matériau utilisé a été synthétisé au laboratoire et soigneusement purifié $\left(T_{\mathrm{SN}}=20^{\circ} \mathrm{C}, T_{\mathrm{NI}}=46^{\circ} \mathrm{C}\right)$.

Les spectres ont été obtenus à l'aide d'un spectromètre Raman laser CODERG type PH1 permettant une résolution de l'ordre de $\pm 2 \mathrm{~cm}^{-1}$. La gamme de température accessible à l'aide du dispositif commercial est comprise entre $77 \mathrm{~K}$ et l'ambiante. Afin de pouvoir réaliser des mesures au-dessus de la température ambiante, nous avons mis au point une cellule chauffante adaptable à cet appareillage.

Les mesures de température sont réalisées avec une précision de $\pm 0,1^{\circ}$ dans le domaine des basses températures et de $\pm 1^{\circ}$ au-dessus de l'ambiante. Afin de ne pas créer de gradient thermique au sein de l'échantillon, la puissance du laser a été limitée à $250 \mathrm{~mW}$ et après chaque enregistrement nous avons vérifié que les caractéristiques du matériau n'avaient pas été modifiées.

2. Résultats et discussion. - 2.1 ETUDE DU MBBA A L'ÉTAT SOLIDE. - Suivant les auteurs, le MBBA peut se présenter sous deux [10] ou trois [11] phases à l'état solide. Notre étude par spectroscopie Raman, complétée par une observation au microscope polarisant, montre d'une façon certaine qu'il existe trois phases solides A, B et $\mathbf{C}$ dont les spectres caractéristiques sont représentés sur la figure 1 .

La forme A est obtenue par refroidissement brutal de la phase nématique; elle évolue ensuite spontanément vers la phase $B$.

$\mathrm{Si}$ on maintient la forme $\mathrm{B}$ à une température voisine de la température de transition NématiqueSolide, on obtient la forme $\mathrm{C}$ très bien cristallisée. Il est vraisemblable que les formes cristallines que nous avons appelées $B$ et $C$ correspondent respectivement aux espèces métastables et stables décrites par J. Mayer et coll. [10], la forme A, peu cristalline d'apparence, à un verre de même nature que celui décrit par Shinoda et coll. [11].

Les spectres de la figure 1 mettent en évidence l'analogie entre les espèces $B$ et $C$.

La modification du spectre dans le domaine des basses régions $\left(175 \mathrm{~cm}^{-1}-300 \mathrm{~cm}^{-1}\right.$ et $800 \mathrm{~cm}^{-1}$. $1000 \mathrm{~cm}^{-1}$ ) montre que la forme $A$ présente une différence structurale au niveau de la chaîne : on peut l'attribuer à l'existence d'isomères trans et gauche gelés alors que dans les espèces $\mathrm{B}$ et $\mathrm{C}$, la chaîne est vraisemblablement entièrement trans comme dans toute phase solide [5].

2.2 Etude De L'état Nématique. - On peut constater (Fig. 1) que toutes les raies des formes B et $\mathrm{C}$ se retrouvent dans le spectre à l'état nématique. Seules, certaines raies de la forme A subsistent (raies à 824 et $311 \mathrm{~cm}^{-1}$ par exemple).

Une raie nouvelle apparaît à $781 \mathrm{~cm}^{-1}$, correspondant à un isomère gauche qui n'existait pas dans les phases solides.
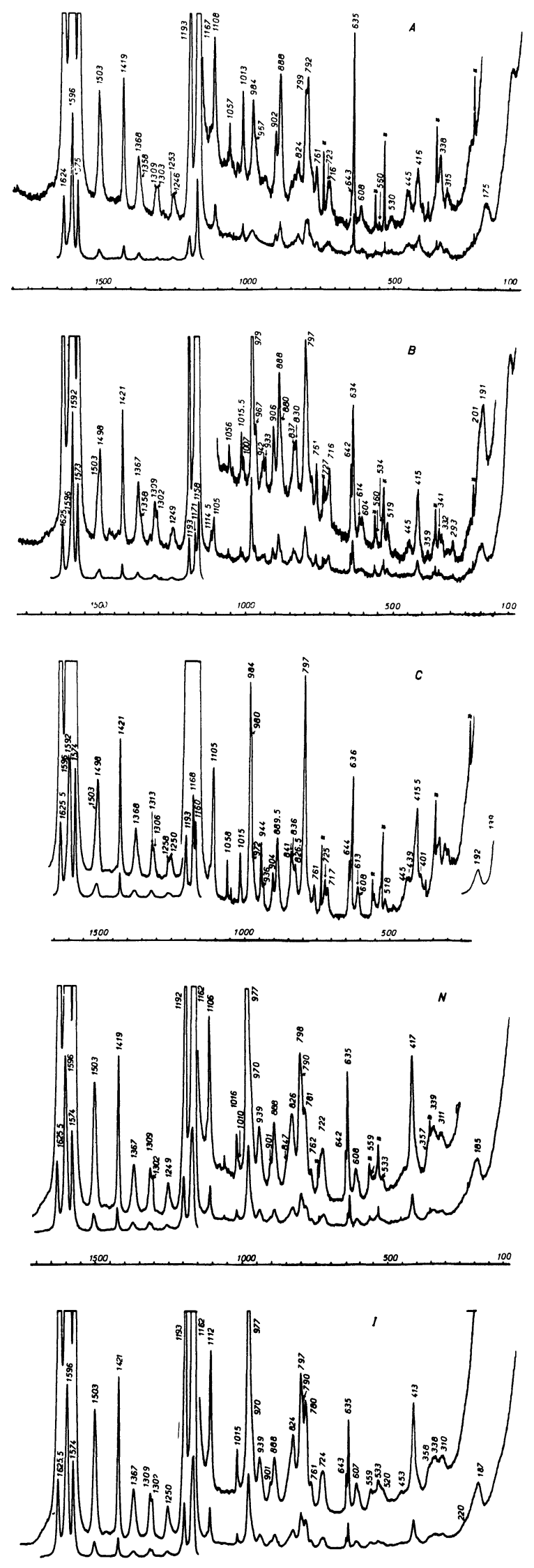

FIG. 1. - Spectres Raman de 2000 à $100 \mathrm{~cm}^{-1}$ du MBBA solide forme $A, B$ et $C$, nématique (N) et liquide isotrope (I): Les raies marquées d'un * ne sont pas attribuables à l'échantillon. 
2.3 ETUDE DE L'ÉTAT LIQUIDE. - Il n'apparaît pas de raies nouvelles par rapport à l'état nématique mais il faut noter des variations importantes d'intensité des raies à 781 et $311 \mathrm{~cm}^{-1}$ principalement. Nous pouvons donc dire que tous les isomères présents dans la phase liquide existent aussi dans la phase nématique avec, dans certains cas, des proportions modifiées.

2.4 Discussion. - Afin de suivre le processus de fusion de la chaîne, nous avons porté en fonction de la température, la variation de l'intensité relative des raies à $781 \mathrm{~cm}^{-1}$ et $311 \mathrm{~cm}^{-1}$ (qui apparaissent à la transition Solide $(\mathrm{B}$ ou $\mathrm{C}) \rightarrow$ Nématique) mesurée par rapport à l'intensité des raies qui leur sont adjacentes à 798 et $339 \mathrm{~cm}^{-1}$ (Fig. 2). Notons qu'en prenant comme références des raies correspondant aux noyaux benzéniques (par exemple à 1015 et $635 \mathrm{~cm}^{-1}$ ) on obtient des résultats identiques à ceux de la figure 2 . Les deux séries de points se placent sur une même courbe qui indique que la fusion de la chaîne s'effectue en deux temps; les modifications les plus importantes interviennent lors de la transition Solide-Nématique. Il faut noter en outre, l'existence d'effets prétransitionnels particulièrement nets au voisinage de la transition Liquide Isotrope $\rightarrow$ Nématique. Une attribution de chacune des raies aux divers isomères trans et gauches permettra de mesurer directement le paramètre d'ordre au niveau de chacune des rotules de la chaîne, le pourcentage relatif d'isomères étant lié au rapport des intensités des raies correspondantes.

Dans ce but, un calcul des modes normaux de vibration est actuellement en voie d'acheminement et fera l'objet d'un prochain article.

Ce premier travail montre les possibilités de la spectroscopie Raman pour éclaircir les problèmes de conformation moléculaire au sein des phases mésomorphes. Couplée à des calculs permettant une attribution des raies, cette technique nous permettra

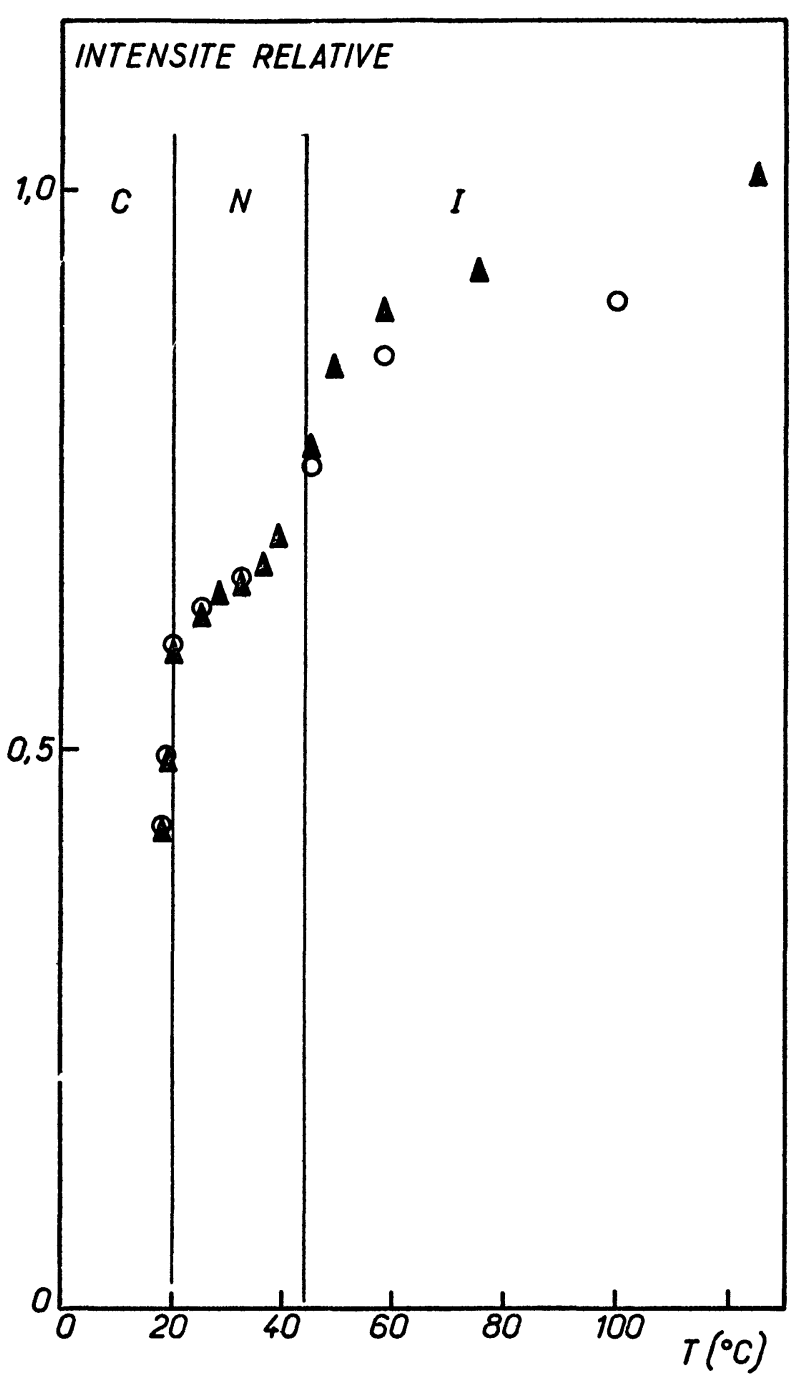

FIG. 2. - Variation de l'intensité relative en fonction de la température des raies du MBBA à $781 \mathrm{~cm}^{-1}(\Delta)$ et $339 \mathrm{~cm}^{-1}(O)$.

de suivre l'évolution de la conformation d'une chaîne en fonction de la température et de tenter de relier les propriétés moléculaires aux propriétés macroscopiques des mésophases.

\section{Bibliographie}

[1] McMillan, W. L., Phys. Rev. A 4 (1971) 1238.

[2] Marčelua, S., Solid State Commun. 13 (1973) 759.

[3] Malthete, J., LeclercQ, M., Dvolaitzky, M., Gabard, J., Billard, J., Pontikis, V. et Jacques, J., Mol. Cryst. Liqu. Cryst. 23 (1973) 215.

[4] Charvolin, J., Deloche, B., Liebert, L. et Strzelecki, L., Soumis au J. Physique.

[5] Vergoten, G., Proceedings of the third International Conference on Raman Spectroscopy, p. 219 (1972) - Thèse Lille (1973).
Billard, J., Delhaye, M., Merlin, J. G. et Vergoten, G., C.R. Hebd. Séan. Acad. Sci. 273B (1971) 1105.

[6] Bulkin, B. J. et Prochaska, F. T., J. Chem. Phys. 54 (1971) 635.

[7] Schnur, J. M., Mol. Cryst. Liqu. Cryst. 23 (1973) 155.

[8] Mizushima, S., Structure of molecules and internal rotation (Academic Press. Inc. New York) 1954.

[9] SNyder, R. G., J. Chem. Phys. 47 (1967) 1316.

[10] Mayer, J., Waluga, T. et Janik, J. A., Phys. Lett. 41 A (1972) 102.

[11] Shinoda, T., Maeda, Y., Enokido, H., 8th Japonese Calorymetry Conf. 1972, Okayana. 